Internat. J. Math. \& Math. Sci.

Vol. 24, No. 7 (2000) 449-459

S0161171200002398

(C) Hindawi Publishing Corp.

\title{
MATRIX-VARIATE BETA DISTRIBUTION
}

\author{
ARJUN K. GUPTA and DAYA K. NAGAR
}

(Received 24 September 1997)

\begin{abstract}
We propose matrix-variate beta type III distribution. Several properties of this distribution including Laplace transform, marginal distribution and its relationship with matrix-variate beta type I and type II distributions are also studied.
\end{abstract}

Keywords and phrases. Matrix variate, beta distribution, zonal polynomial, transformation, Laplace transform.

2000 Mathematics Subject Classification. Primary 62H10; Secondary 62E15.

1. Introduction. The random variable $u$ with the probability density function (pdf)

$$
(\beta(a, b))^{-1} u^{a-1}(1-u)^{b-1}, \quad 0<u<1,
$$

where $a>0$ and $b>0$, is said to have a beta type I distribution with parameters $(a, b)$. The random variable $v$ with pdf,

$$
(\beta(a, b))^{-1} v^{a-1}(1+v)^{-(a+b)}, \quad v>0,
$$

where $a>0$ and $b>0$, is said to have beta type II distribution with parameters $(a, b)$. Since (1.2) can be obtained from (1.1) by the transformation $v=u /(1-u)$, some authors call the distribution of $v$ an "inverted beta distribution."

The matrix variate generalizations of (1.1) and (1.2) are given as follows (see [1, 3, $4,6,11])$.

DEFINITION 1.1. A $p \times p$ random symmetric positive definite matrix $U$ is said to have a matrix-variate beta type I distribution with parameters $(a, b)$, denoted as $U \sim$ $B_{p}^{I}(a, b)$, if its pdf is given by

$$
\left(\beta_{p}(a, b)\right)^{-1} \operatorname{det}(U)^{a-(p+1) / 2} \operatorname{det}\left(I_{p}-U\right)^{b-(p+1) / 2}, \quad 0<U<I_{p},
$$

where $a>(p-1) / 2, b>(p-1) / 2$, and $\beta_{p}(a, b)$ is the multivariate beta function given by

$$
\beta_{p}(a, b)=\frac{\Gamma_{p}(a) \Gamma_{p}(b)}{\Gamma_{p}(a+b)}
$$

where

$$
\Gamma_{p}(a)=\pi^{p(p-1) / 4} \prod_{j=1}^{p} \Gamma\left(a-\frac{j-1}{2}\right), \quad \operatorname{Re}(a)>\frac{p-1}{2} .
$$


DEFINITION 1.2. A $p \times p$ random symmetric positive definite matrix $V$ is said to have a matrix-variate beta type II distribution with parameters $(a, b)$, denoted as $V \sim$ $B_{p}^{I I}(a, b)$, if its pdf is given by

$$
\left(\beta_{p}(a, b)\right)^{-1} \operatorname{det}(V)^{a-(p+1) / 2} \operatorname{det}\left(I_{p}+V\right)^{-(a+b)}, \quad V>0,
$$

where $a>(p-1) / 2, b>(p-1) / 2$, and $\beta_{p}(a, b)$ is the multivariate beta function.

As in the univariate case, the density (1.6) can be obtained from (1.3) by transforming $U=\left(I_{p}+V\right)^{-1} V$, together with the Jacobian $J(U \rightarrow V)=\operatorname{det}\left(I_{p}+V\right)^{-(p+1)}$.

The matrix-variate beta type I and type II distributions have been studied by many authors, e.g., see [7, 9, 10, 13, 14].

In this paper, a new matrix-variate beta distribution has been defined. We call it "Matrix-variate beta type III" distribution, which is then derived by using matrix transformation. Several properties of this distribution and its relationship with matrixvariate beta type I and type II distributions have also been studied.

2. Density function. First, we define the matrix-variate beta distribution of type III.

DEFINITION 2.1. A $p \times p$ random symmetric positive definite matrix $W$ is said to have a matrix-variate beta type III distribution with parameters $(a, b)$, denoted as $W \sim B_{p}^{I I I}(a, b)$, if its pdf is given by

$$
\begin{aligned}
& 2^{p b}\left(\beta_{p}(a, b)\right)^{-1} \operatorname{det}(W)^{b-(p+1) / 2} \\
& \quad \times \operatorname{det}\left(I_{p}-W\right)^{a-(p+1) / 2} \operatorname{det}\left(I_{p}+W\right)^{-(a+b)}, \quad 0<W<I_{p},
\end{aligned}
$$

where $a>(p-1) / 2, b>(p-1) / 2$, and $\beta_{p}(a, b)$ is the multivariate beta function.

For $p=1$, the beta type III density is given by

$$
2^{b}(\beta(a, b))^{-1} w^{b-1}(1-w)^{a-1}(1+w)^{-(a+b)}, \quad 0<w<1,
$$

and in this case we write $w \sim B^{I I I}(a, b)$.

By means of a bilinear transformation of the random matrix $U$, the matrix-variate beta type III distribution is generated as in the following theorem.

ThEOREM 2.1. Let $U \sim B_{p}^{I}(a, b)$. Define $W=\left(I_{p}+U\right)^{-1}\left(I_{p}-U\right)$. Then $W \sim B_{p}^{I I I}(a, b)$.

Proof. Making the transformation $W=\left(I_{p}+U\right)^{-1}\left(I_{p}-U\right)$ with the Jacobian [12] in the pdf (1.3) of $U, J(U \rightarrow W)=2^{p(p+1) / 2}\left(I_{p}+W\right)^{-(p+1)}$, we get the desired result.

From the density of beta type III matrix it is apparent that

$$
\int_{0<W<I_{p}} \begin{aligned}
& \operatorname{det}(W)^{b-(p+1) / 2} \operatorname{det}\left(I_{p}-W\right)^{a-(p+1) / 2} \\
& \times \operatorname{det}\left(I_{p}+W\right)^{-(a+b)} d W=2^{-p b} \beta_{p}(a, b) .
\end{aligned}
$$


The cumulative distribution function (cdf) of $W$ is obtained as

$$
\begin{gathered}
P(W<\Omega)=2^{p b}\left(\beta_{p}(a, b)\right)^{-1} \int_{0<W<\Omega} \operatorname{det}(W)^{b-(p+1) / 2} \operatorname{det}\left(I_{p}-W\right)^{a-(p+1) / 2} \\
\times \operatorname{det}\left(I_{p}+W\right)^{-(a+b)} d W .
\end{gathered}
$$

For evaluating the above integral, we use the following results involving zonal polynomials $[2,5,8]$ :

$$
\begin{gathered}
\operatorname{det}\left(I_{p}-W\right)^{a-(p+1) / 2}=\sum_{k=0}^{\infty} \sum_{\kappa} \frac{(-a+(p+1) / 2)_{\kappa}}{k !} C_{K}(W), \\
\operatorname{det}\left(I_{p}+W\right)^{-(a+b)}=\sum_{t=0}^{\infty} \sum_{\tau} \frac{(a+b)_{\tau}}{t !}(-1)^{t} C_{\tau}(W), \\
C_{K}(W) C_{\tau}(W)=\sum_{\delta} g_{\kappa, \tau}^{\delta} C_{\delta}(W), \\
\int_{0<W<I_{p}} \operatorname{det}(W)^{a-(p+1) / 2} \operatorname{det}\left(I_{p}-W\right)^{b-(p+1) / 2} C_{\kappa}(R W) d W \\
=\beta_{p}(a, b) \frac{(a)_{\kappa}}{(a+b)_{\kappa}} C_{K}(R), \quad \operatorname{Re}(a)>\frac{p-1}{2}, \operatorname{Re}(b)>\frac{p-1}{2},
\end{gathered}
$$

where $\kappa=\left(k_{1}, \ldots, k_{p}\right), k_{1} \geq \cdots \geq k_{p} \geq 0, k_{1}+\cdots+k_{p}=k, \tau=\left(t_{1}, \ldots, t_{p}\right), t_{1} \geq \cdots \geq$ $t_{p} \geq 0, t_{1}+\cdots+t_{p}=t, \delta=\left(d_{1}, \ldots, d_{p}\right), d_{1} \geq \cdots \geq d_{p} \geq 0, d_{1}+\cdots+d_{p}=d=k+t$, and $g_{\kappa, \tau}^{\delta}$ is the coefficient of $C_{\delta}(W)$ in $C_{K}(W) C_{\tau}(W)$. Substituting (2.5) and (2.6) in (2.4) and subsequently using (2.7), we obtain

$$
\begin{aligned}
P(W<\Omega)= & 2^{p b}\left(\beta_{p}(a, b)\right)^{-1} \sum_{k=0}^{\infty} \sum_{t=0}^{\infty} \sum_{\kappa} \sum_{\tau} \frac{(-a+(p+1) / 2)_{\kappa}}{k !} \\
& \times \frac{(a+b)_{\tau}}{t !}(-1)^{t} \sum_{\delta} g_{\kappa, \tau}^{\delta} \int_{0<W<\Omega} \operatorname{det}(W)^{b-(p+1) / 2} C_{\delta}(W) d W .
\end{aligned}
$$

Now substituting $X=\Omega^{-1 / 2} W \Omega^{-1 / 2}$ with the Jacobian $J(W \rightarrow X)=\operatorname{det}(\Omega)^{(p+1) / 2}$ in the above integral, we get

$$
\begin{aligned}
P(W<\Omega)= & 2^{p b}\left(\beta_{p}(a, b)\right)^{-1} \operatorname{det}(\Omega)^{b} \sum_{k=0}^{\infty} \sum_{t=0}^{\infty} \sum_{\kappa} \sum_{\tau} \frac{(-a+(p+1) / 2)_{\kappa}}{k !} \\
& \times \frac{(a+b)_{\tau}}{t !}(-1)^{t} \sum_{\delta} g_{\kappa, \tau}^{\delta} \int_{0<X<I_{p}} \operatorname{det}(X)^{b-(p+1) / 2} C_{\delta}(\Omega X) d X \\
= & 2^{p b} \frac{\Gamma_{p}(a+b) \Gamma_{p}[(p+1) / 2]}{\Gamma_{p}(a) \Gamma_{p}[b+(p+1) / 2]} \operatorname{det}(\Omega)^{b} \sum_{k=0}^{\infty} \sum_{t=0}^{\infty} \sum_{\kappa} \sum_{\tau} \frac{(-a+(p+1) / 2)_{\kappa}}{k !} \\
& \times \frac{(a+b)_{\tau}}{t !}(-1)^{t} \sum_{\delta} g_{\kappa, \tau}^{\delta} \frac{(b)_{\delta}}{(b+(p+1) / 2)_{\delta}} C_{\delta}(\Omega),
\end{aligned}
$$

where the last step has been obtained by using (2.8). 
The Laplace transform of the density of $W$ is

$$
\begin{aligned}
L(Z)=2^{p b}\left(\beta_{p}(a, b)\right)^{-1} \int_{0<W<I_{p}} & \operatorname{etr}(-Z W) \operatorname{det}(W)^{b-(p+1) / 2} \\
\quad & \quad \operatorname{det}\left(I_{p}-W\right)^{a-(p+1) / 2} \operatorname{det}\left(I_{p}+W\right)^{-(a+b)} d W,
\end{aligned}
$$

where $Z(p \times p)=\left(\left(1+\delta_{i j}\right) z_{i j} / 2\right)$. Now, using the expansions

$$
\begin{aligned}
\operatorname{det}\left(I_{p}+W\right)^{-(a+b)} & =2^{-p(a+b)} \operatorname{det}\left(I_{p}-\frac{I_{p}-W}{2}\right)^{-(a+b)} \\
& =2^{-p(a+b)} \sum_{t=0}^{\infty} \sum_{\tau} \frac{(a+b)_{\tau}}{t !}\left(\frac{1}{2}\right)^{t} C_{\tau}\left(I_{p}-W\right), \\
\operatorname{etr}(-Z W) & =\operatorname{etr}(-Z) \operatorname{etr}\left\{Z\left(I_{p}-W\right)\right\}=\operatorname{etr}(-Z) \sum_{k=0}^{\infty} \sum_{\kappa} \frac{1}{k !} C_{\kappa}\left(Z\left(I_{p}-W\right)\right),
\end{aligned}
$$

in (2.11), we obtain

$$
L(Z)=2^{-p a}\left(\beta_{p}(a, b)\right)^{-1} \operatorname{etr}(-Z) \sum_{k=0}^{\infty} \sum_{t=0}^{\infty} \sum_{\kappa} \sum_{\tau} \frac{1}{k !} \frac{(a+b)_{\tau}}{2^{t} t !} \Phi(Z),
$$

where

$$
\begin{aligned}
\Phi(Z) & =\int_{0<W<I_{p}} \operatorname{det}(W)^{b-(p+1) / 2} \operatorname{det}\left(I_{p}-W\right)^{a-(p+1) / 2} C_{K}\left(Z\left(I_{p}-W\right)\right) C_{\tau}\left(I_{p}-W\right) d W \\
& =\int_{0<X<I_{p}} \operatorname{det}\left(I_{p}-X\right)^{b-(p+1) / 2} \operatorname{det}(X)^{a-(p+1) / 2} C_{K}(Z X) C_{\tau}(X) d X
\end{aligned}
$$

Since $\Phi(Z)=\Phi\left(H^{\prime} Z H\right), H \in O(p)$, integrating out $H$ in $\Phi\left(H^{\prime} Z H\right)$ using [5, equation 23], we have

$$
\Phi(Z)=\frac{C_{K}(Z)}{C_{K}\left(I_{p}\right)} \int_{0<X<I_{p}} \operatorname{det}\left(I_{p}-X\right)^{b-(p+1) / 2} \operatorname{det}(X)^{a-(p+1) / 2} C_{K}(X) C_{\tau}(X) d X .
$$

Now using (2.7), and integrating $X$ using (2.8), we obtain

$$
\Phi(Z)=\frac{C_{K}(Z)}{C_{\kappa}\left(I_{p}\right)} \sum_{\delta} g_{\kappa, \tau}^{\delta} \frac{(a)_{\delta}}{(a+b)_{\delta}} \frac{\Gamma_{p}(a) \Gamma_{p}(b)}{\Gamma_{p}(a+b)} C_{\delta}\left(I_{p}\right) .
$$

Substituting (2.16) in (2.13), we finally obtain

$$
L(Z)=2^{-p a} \operatorname{etr}(-Z) \sum_{k=0}^{\infty} \sum_{t=0}^{\infty} \sum_{\kappa} \sum_{\tau} \frac{1}{k !} \frac{(a+b)_{\tau}}{2^{t} t !} \frac{C_{K}(Z)}{C_{\kappa}\left(I_{p}\right)} \sum_{\delta} g_{\kappa, \tau}^{\delta} \frac{(a)_{\delta}}{(a+b)_{\delta}} C_{\delta}\left(I_{p}\right) .
$$

3. Properties. In this section, we study some properties of the random matrix distributed as matrix-variate beta type III. 
THEOREM 3.1. Let $W \sim B_{p}^{I I I}(a, b)$ and $A(p \times p)$ be a constant nonsingular matrix. Then the density of $X=A W A^{\prime}$ is

$$
\begin{aligned}
& 2^{p b}\left(\beta_{p}(a, b)\right)^{-1} \operatorname{det}\left(A A^{\prime}\right)^{(p+1) / 2} \operatorname{det}(X)^{b-(p+1) / 2} \\
& \quad \times \operatorname{det}\left(A A^{\prime}-X\right)^{a-(p+1) / 2} \operatorname{det}\left(A A^{\prime}+X\right)^{-(a+b)}, \quad 0<X<A A^{\prime} .
\end{aligned}
$$

Proof. Making the transformation $X=A W A^{\prime}$ with the Jacobian $J(W \rightarrow X)=$ $\operatorname{det}(A)^{-(p+1)}$ in the pdf (2.1) of $W$, the density of $X$ is obtained as

$$
\begin{aligned}
& 2^{p b}\left(\beta_{p}(a, b)\right)^{-1} \operatorname{det}\left(A A^{\prime}\right)^{(p+1) / 2} \operatorname{det}(X)^{b-(p+1) / 2} \\
& \quad \times \operatorname{det}\left(A A^{\prime}-X\right)^{a-(p+1) / 2} \operatorname{det}\left(A A^{\prime}+X\right)^{-(a+b)}, \quad 0<X<A A^{\prime} .
\end{aligned}
$$

which is the desired result.

We will write $X \sim B_{p}^{I I I}\left(a, b ; A A^{\prime}\right)$. In the next theorem, it is shown that the matrixvariate beta distribution of type III is orthogonally invariant.

THEOREM 3.2. Let $W \sim B_{p}^{I I I}(a, b)$ and $H(p \times p)$ be an orthogonal matrix, whose elements are either constants or random variables distributed independently of $W$. Then, the distribution of $W$ is invariant under the transformation $W \rightarrow H W H^{\prime}$, and does not depend on $H$.

Proof. First, let $H$ be a constant orthogonal matrix. Then, from Theorem 3.1, $H W H^{\prime} \sim B_{p}^{I I I}(a, b)$ since $H H^{\prime}=I_{p}$. If, however, $H$ is a random orthogonal matrix, then the conditional distribution of $H U H^{\prime} \mid H \sim B_{p}^{I I I}(a, b)$. Since this distribution does not depend on $H, H W H^{\prime} \sim B_{p}^{I I I}(a, b)$.

The relationship between beta type I, type II, and type III matrices is now exhibited. First, we derive the density of $W^{-1}$.

THEOREM 3.3. Let $W \sim B_{p}^{I I I}(a, b)$. Then the density of $Y=W^{-1}$ is

$$
2^{p b}\left(\beta_{p}(a, b)\right)^{-1} \operatorname{det}\left(Y-I_{p}\right)^{a-(p+1) / 2} \operatorname{det}\left(I_{p}+Y\right)^{-(a+b)}, \quad Y>I_{p} .
$$

Proof. Making the transformation $Y=W^{-1}$ with the Jacobian $J(W \rightarrow Y)=$ $\operatorname{det}(Y)^{-(p+1)}$, in the density of $W$ the result follows.

The density derived above may be called the inverse beta type III. From Theorem 3.3, it is clear that if $W \sim B_{p}^{I I I}(a, b)$, then $W^{-1}$ does not follow beta type III distribution.

THEOREM 3.4. (i) Let $U \sim B_{p}^{I}(a, b)$ and $W=\left(I_{p}+U\right)^{-1}\left(I_{p}-U\right)$, then $W \sim B_{p}^{I I I}(a, b)$. (ii) Similarly, if $W \sim B_{p}^{I I I}(a, b)$ and $U=\left(I_{p}+W\right)^{-1}\left(I_{p}-W\right)$, then $U \sim B_{p}^{I}(a, b)$.

Proof. Part (i) is proved in Theorem 2.1. Since $W=\left(I_{p}+U\right)^{-1}\left(I_{p}-U\right)$, the Jacobian of transformation is $J(W \rightarrow U)=2^{p(p+1) / 2} \operatorname{det}\left(I_{p}+U\right)^{-(p+1)}$. Now, making the substitution in the density of $W$ given by (2.1) the result (ii) follows.

THEOREM 3.5. (i) Let $V \sim B_{p}^{I I}(a, b)$ and $W=\left(I_{p}+2 V\right)^{-1}$, then $W \sim B_{p}^{I I I}(a, b)$.

(ii) Similarly, if $W \sim B_{p}^{I I I}(a, b)$ and $V=\left(I_{p}-W\right) W^{-1} / 2$, then $V \sim B_{p}^{I I}(a, b)$.

The marginal distributions of $W$ is given in the following. 
THEOREM 3.6. Let $W=\left(\begin{array}{cc}W_{11} & W_{12} \\ W_{21} & W_{22}\end{array}\right), W_{11}(q \times q)$, and $W_{22 \cdot 1}=W_{22}-W_{21} W_{11}^{-1} W_{12}$. If $W \sim B_{p}^{I I I}(a, b)$, then $W_{22 \cdot 1} \sim B_{p-q}^{I I I}(a, b-q / 2)$.

Proof. From the partition of $W$, we have

$$
\begin{aligned}
\operatorname{det}(W) & =\operatorname{det}\left(W_{11}\right) \operatorname{det}\left(W_{22 \cdot 1}\right), \\
\operatorname{det}\left(I_{p}-W\right) & =\operatorname{det}\left(I_{q}-W_{11}\right) \operatorname{det}\left(I_{p-q}-W_{22 \cdot 1}-W_{21} W_{11}^{-1}\left(I_{q}-W_{11}\right)^{-1} W_{12}\right), \\
\operatorname{det}\left(I_{p}+W\right) & =\operatorname{det}\left(I_{q}+W_{11}\right) \operatorname{det}\left(I_{p-q}+W_{22 \cdot 1}+W_{21} W_{11}^{-1}\left(I_{q}+W_{11}\right)^{-1} W_{12}\right) .
\end{aligned}
$$

Now making the transformation $W_{11}=W_{11}, X=W_{21} W_{11}^{-1 / 2}$, and $W_{22 \cdot 1}=W_{22}-$ $W_{21} W_{11}^{-1} W_{12}=W_{22}-X X^{\prime}$ with Jacobian $J\left(W_{11}, W_{22}, W_{21} \rightarrow W_{11}, W_{22 \cdot 1}, X\right)=$ $\operatorname{det}\left(W_{11}\right)^{(p-q) / 2}$ and substituting (3.4), (3.5), and (3.6) in the density of $W$, we get the joint density of $W_{11}, W_{22 \cdot 1}$, and $X$ as follows:

$$
\begin{aligned}
& 2^{p b}\left(\beta_{p}(a, b)\right)^{-1} \operatorname{det}\left(W_{11}\right)^{b-(q+1) / 2} \operatorname{det}\left(I_{q}-W_{11}\right)^{a-(p+1) / 2} \operatorname{det}\left(I_{q}+W_{11}\right)^{-(a+b)} \\
& \quad \times \operatorname{det}\left(W_{22 \cdot 1}\right)^{b-(p+1) / 2} \operatorname{det}\left(I_{p-q}-W_{22 \cdot 1}\right)^{a-(p+1) / 2} \operatorname{det}\left(I_{p-q}+W_{22 \cdot 1}\right)^{-(a+b)} \\
& \quad \times \operatorname{det}\left(I_{p-q}-\left(I_{p-q}-W_{22 \cdot 1}\right)^{-1} X\left(I_{q}-W_{11}\right)^{-1} X^{\prime}\right)^{a-(p+1) / 2} \\
& \quad \times \operatorname{det}\left(I_{p-q}+\left(I_{p-q}+W_{22 \cdot 1}\right)^{-1} X\left(I_{q}+W_{11}\right)^{-1} X^{\prime}\right)^{-(a+b)} .
\end{aligned}
$$

Substituting $Y=\left(I_{p-q}-W_{22 \cdot 1}\right)^{-1 / 2} X\left(I_{q}-W_{11}\right)^{-1 / 2}$ with Jacobian $J(X \rightarrow Y)=$ $\operatorname{det}\left(I_{p-q}-W_{22 \cdot 1}\right)^{q / 2} \operatorname{det}\left(I_{q}-W_{11}\right)^{(p-q) / 2}$ and integrating $Y$, we get the joint density of $W_{11}$ and $W_{22 \cdot 1}$ as

$$
\begin{aligned}
& 2^{p b}\left(\beta_{p}(a, b)\right)^{-1} \operatorname{det}\left(W_{11}\right)^{b-(q+1) / 2} \operatorname{det}\left(I_{q}-W_{11}\right)^{a-(q+1) / 2} \operatorname{det}\left(I_{q}+W_{11}\right)^{-(a+b)} \\
& \times \operatorname{det}\left(W_{22 \cdot 1}\right)^{b-(p+1) / 2} \operatorname{det}\left(I_{p-q}-W_{22 \cdot 1}\right)^{a-(p-q+1) / 2} \operatorname{det}\left(I_{p-q}+W_{22 \cdot 1}\right)^{-(a+b)} g(A, B),
\end{aligned}
$$

where

$$
\begin{aligned}
& g(A, B)=\int_{I_{p-q}-Y Y^{\prime}>0} \operatorname{det}\left(I_{p-q}-Y Y^{\prime}\right)^{a-(p+1) / 2} \operatorname{det}\left(I_{p-q}+A Y B Y^{\prime}\right)^{-(a+b)} d Y \\
& =\int_{0<Z<I_{p-q}} \int_{Y Y^{\prime}=Z} \operatorname{det}\left(I_{p-q}-Y Y^{\prime}\right)^{a-(p+1) / 2}{ }_{1} F_{0}^{(q)}\left(a+b ;-Y^{\prime} A Y B\right) d Y d Z \text {, for } p-q \leq q, \\
& =\int_{0<Z<I_{q}} \int_{Y^{\prime} Y=Z} \operatorname{det}\left(I_{q}-Y^{\prime} Y\right)^{a-(p+1) / 2}{ }_{1} F_{0}^{(p-q)}\left(a+b ;-A Y B Y^{\prime}\right) d Y d Z, \text { for } p-q>q,
\end{aligned}
$$

with $A=\left(I_{p-q}-W_{22 \cdot 1}\right)^{1 / 2}\left(I_{p-q}+W_{22 \cdot 1}\right)^{-1}\left(I_{p-q}-W_{22 \cdot 1}\right)^{1 / 2}$ and $B=\left(I_{q}-W_{11}\right)^{1 / 2}\left(I_{q}+\right.$ $\left.W_{11}\right)^{-1}\left(I_{q}-W_{11}\right)^{1 / 2}$. Since $g(A, B)=g\left(A, H^{\prime} B H\right), H \in O(q)$, by integrating $H$ in $g\left(A, H^{\prime} B H\right)$, we obtain 


$$
\begin{aligned}
& g(A, B)=\int_{0<Z<I_{p-q}} \int_{Y Y^{\prime}=Z} \operatorname{det}\left(I_{p-q}-Y Y^{\prime}\right)^{a-(p+1) / 2}{ }_{1} F_{0}^{(q)}\left(a+b ;-A Y Y^{\prime}, B\right) d Y d Z \\
& =\frac{\pi^{q(p-q) / 2}}{\Gamma_{p-q}(q / 2)} \int_{0<Z<I_{p-q}} \operatorname{det}(Z)^{(q-p+q-1) / 2} \operatorname{det}\left(I_{p-q}-Z\right)^{a-(p+1) / 2}{ }_{1} F_{0}^{(q)}(a+b ;-A Z, B) d Z \\
& =\frac{\pi^{q(p-q) / 2}}{\Gamma_{p-q}(q / 2)} \cdot \frac{\Gamma_{p-q}(q / 2) \Gamma_{p-q}(a-q / 2)}{\Gamma_{p-q}(a)}{ }_{2} F_{1}^{(q)}\left(\frac{q}{2}, a+b ; a ;-A, B\right) \\
& =\frac{\pi^{q(p-q) / 2} \Gamma_{p-q}(a-q / 2)}{\Gamma_{p-q}(a)}{ }_{2} F_{1}^{(q)}\left(\frac{q}{2}, a+b ; a ;-A, B\right) .
\end{aligned}
$$

Substituting $g(A, B)$ in (3.8), we get the joint density of $W_{11}$ and $W_{22 \cdot 1}$ as

$$
\begin{aligned}
2^{q b} & \left(\beta_{q}(a, b)\right)^{-1} \operatorname{det}\left(W_{11}\right)^{b-(q+1) / 2} \operatorname{det}\left(I_{q}-W_{11}\right)^{a-(q+1) / 2} \\
& \times \operatorname{det}\left(I_{q}+W_{11}\right)^{-(a+b)} 2^{(p-q)(b-q / 2)}\left(\beta_{p-q}\left(a, b-\frac{q}{2}\right)\right)^{-1} \operatorname{det}\left(W_{22 \cdot 1}\right)^{b-(p+1) / 2} \\
& \times \operatorname{det}\left(I_{p-q}-W_{22 \cdot 1}\right)^{a-(p-q+1) / 2} \operatorname{det}\left(I_{p-q}+W_{22 \cdot 1}\right)^{-(a+b)} 2^{(p-q) q / 2} \\
& \times{ }_{2} F_{1}^{(q)}\left(\frac{q}{2}, a+b ; a ;-A, B\right) .
\end{aligned}
$$

Clearly $W_{11}$ and $W_{22 \cdot 1}$ are not independent. Integrating $W_{11}$, using

$$
\begin{aligned}
2^{q b} & \int_{0<W_{11}<I_{q}} \operatorname{det}\left(W_{11}\right)^{b-(q+1) / 2} \operatorname{det}\left(I_{q}-W_{11}\right)^{a-(q+1) / 2} \operatorname{det}\left(I_{q}+W_{11}\right)^{-(a+b)} \\
& \times{ }_{2} F_{1}^{(q)}\left(\frac{q}{2}, a+b ; a ;-A, B\right) d W_{11} \\
= & \int_{0<B<I_{q}} \operatorname{det}(B)^{a-(q+1) / 2} \operatorname{det}\left(I_{q}-B\right)^{b-(q+1) / 2}{ }_{2} F_{1}\left(\frac{q}{2}, a+b ; a ;-A, B\right) d B \\
= & \beta_{q}(a, b){ }_{3} F_{2}^{(q)}\left(a, \frac{q}{2}, a+b ; a, a+b ;-A\right) \\
= & \beta_{q}(a, b) \operatorname{det}\left(I_{p-q}+A\right)^{-q / 2} \\
= & 2^{-(p-q) q / 2} \beta_{q}(a, b) \operatorname{det}\left(I_{p-q}+W_{22 \cdot 1}\right)^{q / 2},
\end{aligned}
$$

we get the density of $W_{22 \cdot 1}$. For $q<p-q$, using (3.9) and following similar steps we get the same result.

Alternately, Theorem 3.6 can be proved using the relationship between type II and type III beta matrices. Let $V \sim B_{p}^{I I}(a, b)$ and $V=\left(\begin{array}{ll}V_{11} & V_{12} \\ V_{21} & V_{22}\end{array}\right), V_{11}(q \times q)$ and $V_{11 \cdot 2}=V_{11}-$ $V_{12} V_{22}^{-1} V_{21}$. It is well known that $V_{11 \cdot 2}$ and $V_{22}$ are distributed independently (see [4]), $V_{11 \cdot 2} \sim B_{q}^{I I}(a-(p-q) / 2, b)$ and $V_{22} \sim B_{p-q}^{I I}(a, b-q / 2)$. According to Theorem 3.5(i), if $V \sim B_{p}^{I I}(a, b)$, then $W=\left(I_{p}+2 V\right)^{-1} \sim B_{p}^{I I I}(a, b)$. Furthermore,

$$
W^{-1}=\left(\begin{array}{ll}
W^{11} & W^{12} \\
W^{21} & W^{22}
\end{array}\right)=\left(\begin{array}{cc}
I_{q}+2 V_{11} & 2 V_{12} \\
2 V_{21} & I_{p-q}+2 V_{22}
\end{array}\right) .
$$

That is, $W^{22}((p-q) \times(p-q))=W_{22 \cdot 1}^{-1}=I_{p-q}+2 V_{22}$ and $W_{22 \cdot 1}=\left(I_{q}+2 V_{22}\right)^{-1}$. 
Now, since $V_{22} \sim B_{p-q}^{I I}(a, b-q / 2)$, then $W_{22 \cdot 1}=\left(I_{p-q}+2 V_{22}\right)^{-1} \sim B_{p-q}^{I I I}(a, b-q / 2)$.

The distribution of $\left(A W^{-1} A^{\prime}\right)^{-1}$ where $A(q \times p)$ is a constant matrix of rank $q(\leq p)$, is now derived.

THEOREM 3.7. Let $A(q \times p)$ be a constant matrix of rank $q(\leq p)$. If $W \sim B_{p}^{I I I}(a, b)$, then $\left(A W^{-1} A^{\prime}\right)^{-1} \sim B_{q}^{I I I}\left(a, b-(p-q) / 2 ;\left(A A^{\prime}\right)^{-1}\right)$.

Proof. We write $A=M\left(I_{q} \quad 0\right) G$, where $M(q \times q)$ is nonsingular and $G(p \times p)$ is orthogonal. Now,

$$
\begin{aligned}
& \left.\left(A W^{-1} A^{\prime}\right)^{-1}=\left(\begin{array}{ll}
M\left(I_{q}\right. & 0
\end{array}\right) G W^{-1} G^{\prime}\left(\begin{array}{ll}
I_{q} & 0
\end{array}\right)^{\prime} M^{\prime}\right)^{-1} \\
& =\left(M^{\prime}\right)^{-1}\left[\left(\begin{array}{ll}
I_{q} & 0
\end{array}\right) Y^{-1}\left(\begin{array}{c}
I_{q} \\
0
\end{array}\right)\right]^{-1} M^{-1} \\
& =\left(M^{\prime}\right)^{-1}\left(Y^{11}\right)^{-1} M^{-1} \text {, }
\end{aligned}
$$

where $Y=\left(\begin{array}{ll}Y_{11} & Y_{12} \\ Y_{21} & Y_{22}\end{array}\right)=G W G^{\prime} \sim B_{p}^{I I I}(a, b), Y_{11}(q \times q)$ and $Y^{11}=\left(Y_{11}-Y_{12} Y_{22}^{-1} Y_{21}\right)^{-1}=$ $Y_{11 \cdot 2}^{-1}$. From Theorem 3.6, $Y_{11 \cdot 2} \sim B_{q}^{I I I}(a, b-(p-q) / 2)$ and from Theorem 3.1, $\left(M^{\prime}\right)^{-1} Y_{22 \cdot 1} M^{-1} \sim B_{q}^{I I I}\left(a, b-(p-q) / 2 ;\left(M M^{\prime}\right)^{-1}\right)$. The proof is now completed by observing that $M M^{\prime}=A A^{\prime}$.

COROLLARY 3.8. Let $W \sim B_{p}^{I I I}(a, b)$ and $\boldsymbol{a} \in \mathfrak{R}^{p}, \boldsymbol{a} \neq \mathbf{0}$, then $\boldsymbol{a}^{\prime} \boldsymbol{a} / \boldsymbol{a}^{\prime} W^{-1} \boldsymbol{a} \sim B^{I I I}(a, b-$ $(p-1) / 2)$.

In Corollary 3.8, the distribution of $\boldsymbol{a}^{\prime} \boldsymbol{a} / \boldsymbol{a}^{\prime} W^{-1} \boldsymbol{a}$ does not depend on $\boldsymbol{a}$. Thus, if $\boldsymbol{y}(p \times 1)$ is a random vector, independent of $W$, and $P(\boldsymbol{y} \neq \mathbf{0})=1$, then it follows that $\boldsymbol{y}^{\prime} \boldsymbol{y} / \boldsymbol{y}^{\prime} W^{-1} \boldsymbol{y} \sim B^{I I I}(a, b-(p-1) / 2)$.

THEOREM 3.9. Let $W \sim B_{p}^{I I I}(a, b)$, then

(i) $E\left[\frac{\operatorname{det}(W)^{h}}{\operatorname{det}\left(I_{p}+W\right)^{h}}\right]=\frac{\Gamma_{p}(a+b) \Gamma_{p}(b+h)}{2^{p h} \Gamma_{p}(b)(a+b+h)}, \quad \operatorname{Re}(h)>-b+\frac{p-1}{2}$,

(ii) $E\left[\operatorname{det}(W)^{h}\right]$

$$
=\frac{2^{-p a} \Gamma_{p}(a+b) \Gamma_{p}(b+h)}{\Gamma_{p}(b) \Gamma_{p}(a+b+h)}{ }_{2} F_{1}\left(a, a+b ; a+b+h, \frac{1}{2} I_{p}\right), \quad \operatorname{Re}(h)>-b+\frac{p-1}{2},
$$

(iii) $E\left[\operatorname{det}\left(I_{p}-W\right)^{h}\right]$

$$
=\frac{2^{-p a} \Gamma_{p}(a+b) \Gamma_{p}(a+h)}{\Gamma_{p}(a) \Gamma_{p}(a+b+h)}{ }_{2} F_{1}\left(a+h, a+b ; a+b+h, \frac{1}{2} I_{p}\right), \quad \operatorname{Re}(h)>-a+\frac{p-1}{2},
$$

where ${ }_{2} F_{1}$ is the hypergeometric function of matrix argument.

Proof. (i) From the density of $W$, we have

$$
\begin{aligned}
E\left[\frac{\operatorname{det}(W)^{h}}{\left.\operatorname{det}\left(I_{p}+W\right)^{h}\right]}\right. & =2^{p b}\left(\beta_{p}(a, b)\right)^{-1} \int_{0<W<I_{p}} \operatorname{det}(W)^{b+h-(p+1) / 2} \operatorname{det}\left(I_{p}-W\right)^{a-(p+1) / 2} \\
& \quad \times \operatorname{det}\left(I_{p}+W\right)^{-(a+b+h)} d W \\
& =2^{p b}\left(\beta_{p}(a, b)\right)^{-1} \frac{\beta_{p}(a, b+h)}{2^{p(b+h)}}, \quad \operatorname{Re}(h)>-b+\frac{p-1}{2}
\end{aligned}
$$


Simplifying this last expression using (1.4), we get the desired result.

(ii) From the density of $W$, we have

$$
\begin{aligned}
E\left[\operatorname{det}(W)^{h}\right]= & 2^{p b}\left(\beta_{p}(a, b)\right)^{-1} \\
& \times \int_{0<W<I_{p}} \operatorname{det}(W)^{b+h-(p+1) / 2} \operatorname{det}\left(I_{p}-W\right)^{a-(p+1) / 2} \operatorname{det}\left(I_{p}+W\right)^{-(a+b)} d W
\end{aligned}
$$

Writing $\operatorname{det}\left(I_{p}+W\right)^{-(a+b)}$ in series involving zonal polynomials using (2.12), we obtain

$$
\begin{aligned}
E[ & \left.\operatorname{det}(W)^{h}\right] \\
= & 2^{-p a}\left(\beta_{p}(a, b)\right)^{-1} \sum_{t=0}^{\infty} \sum_{\tau} \frac{(a+b)_{\tau}}{2^{t} t !} \\
& \times \int_{0<W<I_{p}} \operatorname{det}(W)^{b+h-(p+1) / 2} \operatorname{det}\left(I_{p}-W\right)^{a-(p+1) / 2} C_{\tau}\left(I_{p}-W\right) d W \\
= & 2^{-p a} \frac{\beta_{p}(a, b+h)}{\beta_{p}(a, b)} \sum_{t=0}^{\infty} \sum_{\tau} \frac{(a+b)_{\tau}}{s^{t} t !} \cdot \frac{a_{\tau}}{(a+b+h)_{\tau}} C_{T}\left(I_{p}\right), \quad \operatorname{Re}(h)>-b+\frac{p-1}{2},
\end{aligned}
$$

where the integral has been evaluated using (2.8). Finally, simplifying the expression using results on hypergeometric functions $[2,5]$, we get the desired result.

Similarly $E\left[\operatorname{det}\left(I_{p}-W\right)^{h}\right]$ can be derived.

From the density of $W$, we have

$$
\begin{aligned}
E\left[C_{K}(W)\right]=2^{p b}\left(\beta_{p}(a, b)\right)^{-1} \int_{0<W<I_{p}} & C_{K}(W) \operatorname{det}(W)^{b-(p+1) / 2} \\
& \times \operatorname{det}\left(I_{p}-W\right)^{a-(p+1) / 2} \operatorname{det}\left(I_{p}+W\right)^{-(a+b)} d W .
\end{aligned}
$$

Writing $\operatorname{det}\left(I_{p}+W\right)^{-(a+b)}$ in series involving zonal polynomials using (2.6), we obtain

$$
\begin{aligned}
E\left[C_{K}(W)\right]= & 2^{p b}\left(\beta_{p}(a, b)\right)^{-1} \sum_{t=0}^{\infty} \sum_{\tau} \frac{(a+b)_{\tau}}{t !}(-1)^{t} \\
& \times \int_{0<W<I_{p}} C_{\kappa}(W) C_{\tau}(W) \operatorname{det}(W)^{b-(p+1) / 2} \operatorname{det}\left(I_{p}-W\right)^{a-(p+1) / 2} d W \\
= & 2^{p b}\left(\beta_{p}(a, b)\right)^{-1} \sum_{t=0}^{\infty} \sum_{\tau} \frac{(a+b)_{\tau}}{t !}(-1)^{t} \sum_{\delta} g_{\kappa, \tau}^{\delta} \\
& \times \int_{0<W<I_{p}} C_{\delta}(W) \operatorname{det}(W)^{b-(p+1) / 2} \operatorname{det}\left(I_{p}-W\right)^{a-(p+1) / 2} d W \\
= & 2^{p b} \sum_{t=0}^{\infty} \sum_{\tau} \frac{(a+b)_{\tau}}{t !}(-1)^{t} \sum_{\delta} g_{\kappa, \tau}^{\delta} \frac{(b)_{\delta}}{(a+b)_{\delta}} C_{\delta}\left(I_{p}\right),
\end{aligned}
$$

where the last two steps have been obtained using (2.7) and (2.8). 
THEOREM 3.10. (i) Let $U \sim B_{p}^{I}(a, b)$ and $W \sim B_{p}^{I I I}(c, a+b)$ be independent. Then $Y=W^{1 / 2} U W^{1 / 2}, 0<Y<I_{p}$ has the density

$$
\begin{aligned}
2^{p(a+b)} & \left(\beta_{p}(a, b+c)\right)^{-1} \operatorname{det}(Y)^{a-(p+1) / 2} \operatorname{det}\left(I_{p}-Y\right)^{b+c-(p+1) / 2} \\
& \times \operatorname{det}\left(I_{p}+Y\right)^{-(a+b+c)}{ }_{2} F_{1}\left(b, a+b+c ; b+c ;-\left(I_{p}+Y\right)^{-1}\left(I_{p}-Y\right)\right), \quad 0<Y<I_{p} .
\end{aligned}
$$

Further, pdf of $X=\left(I_{p}+Y\right)^{-1}\left(I_{p}-Y\right)=\left(I_{p}+W^{1 / 2} U W^{1 / 2}\right)^{-1}\left(I_{p}-W^{1 / 2} U W^{1 / 2}\right)$ is

$$
\begin{aligned}
2^{p b}\left(\beta_{p}(a, b+c)\right)^{-1} \operatorname{det}(X)^{b+c-(p+1) / 2} \operatorname{det}\left(I_{p}-X\right)^{a-(p+1) / 2} & \\
& \times{ }_{2} F_{1}(b, a+b+c ; b+c ;-X), \quad 0<X<I_{p} .
\end{aligned}
$$

(ii) Let $U \sim B_{p}^{I}(a, b)$ and $V \sim B_{p}^{I}(a+b, c)$ be independent. Then $Y=\left(I_{p}+V^{1 / 2} U V^{1 / 2}\right)^{-1}$ $\times\left(I_{p}-V^{1 / 2} U V^{1 / 2}\right) \sim B_{p}^{I I I}(a, b+c)$.

Proof. The joint density of $U$ and $V$ is

$$
\begin{aligned}
2^{p(a+b)}\left(\beta_{p}\right. & \left.(a, b) \beta_{p}(c, a+b)\right)^{-1} \operatorname{det}(U)^{a-(p+1) / 2} \operatorname{det}\left(I_{p}-U\right)^{b-(p+1) / 2} \\
& \times \operatorname{det}(W)^{a+b-(p+1) / 2} \operatorname{det}\left(I_{p}-W\right)^{c-(p+1) / 2} \\
& \times \operatorname{det}\left(I_{p}+W\right)^{-(a+b+c)}, \quad 0<U<I_{p}, 0<W<I_{p} .
\end{aligned}
$$

Making the transformation $Y=W^{1 / 2} U W^{1 / 2}$ with Jacobian $J(U \rightarrow Y)=\operatorname{det}(W)^{-(p+1) / 2}$ in above, we get the joint density of $Y$ and $W$ as

$$
\begin{aligned}
& 2^{p(a+b)}\left(\beta_{p}(a, b) \beta_{p}(c, a+b)\right)^{-1} \operatorname{det}(Y)^{a-(p+1) / 2} \operatorname{det}(W-Y)^{b-(p+1) / 2} \\
& \quad \times \operatorname{det}\left(I_{p}-W\right)^{c-(p+1) / 2} \operatorname{det}\left(I_{p}+W\right)^{-(a+b+c)}, \quad 0<Y<W<I_{p} .
\end{aligned}
$$

Substituting $Z=\left(I_{p}-Y\right)^{-1 / 2}(W-Y)\left(I_{p}-Y\right)^{-1 / 2}$ with the Jacobian $J(W \rightarrow Z)=$ $\operatorname{det}\left(I_{p}-Y\right)^{(p+1) / 2}$ and integrating $Z$, we get the density of $Y$ as

$$
\begin{aligned}
2^{p(a+b)} & \left(\beta_{p}(a, b) \beta_{p}(c, a+b)\right)^{-1} \operatorname{det}(Y)^{a-(p+1) / 2} \\
& \times \operatorname{det}\left(I_{p}-Y\right)^{b+c-(p+1) / 2} \operatorname{det}\left(I_{p}+Y\right)^{-(a+b+c)} \\
& \times \int_{0<Z<I_{p}} \operatorname{det}(Z)^{b-(p+1) / 2} \operatorname{det}\left(I_{p}-Z\right)^{c-(p+1) / 2} \\
& \times \operatorname{det}\left(I_{p}+\left(I_{p}+Y\right)^{-1}\left(I_{p}-Y\right) Z\right)^{-(a+b+c)} d Z .
\end{aligned}
$$

Integration of $Z$ using [5, equation 48] completes the proof.

(ii) From [6], we have $V^{1 / 2} U V^{1 / 2} \sim B_{p}^{I}(a, b+c)$ and from Theorem 2.1, we have $\left(I_{p}+V^{1 / 2} U V^{1 / 2}\right)^{-1}\left(I_{p}-V^{1 / 2} U V^{1 / 2}\right) \sim B_{p}^{I I I}(a, b+c)$. 
Acknowledgement. This research was supported by the Comité para el Desarrollo de la Investigación, Universidad de Antioquia research grant no. IN91CE.

\section{REFERENCES}

[1] Y. Asoo, On the $\Gamma$-distribution of matrix argument and its related distribution, Natural Science 2 (1969), 1-13, Memoirs of Faculty of Literature and Sciences, Shimane University. Zbl 184.22304.

[2] A. G. Constantine, Some non-central distribution problems in multivariate analysis, Ann. Math. Statist. 34 (1963), 1270-1285. MR 31\#5285. Zbl 123.36801.

[3] A. P. Dawid, Some matrix-variate distribution theory: notational considerations and a Bayesian application, Biometrika 68 (1981), no. 1, 265-274. MR 83m:62083. Zbl 464.62039.

[4] A. K. Gupta and D. K. Nagar, Matrix Variate Distributions, Chapman \& Hall/CRC, Boca Raton, FL, 2000. CMP 1738933.

[5] A. T. James, Distributions of matrix variates and latent roots derived from normal samples, Ann. Math. Statist. 35 (1964), 475-501. MR 31\#5286. Zbl 121.36605.

[6] W. R. Javier and A. K. Gupta, On generalized matric variate beta distributions, Statistics 16 (1985), no. 4, 549-558. MR 86m:62023. Zbl 602.62039.

[7] C. G. Khatri, A note on Mitra's paper "A density-free approach to the matrix variate beta distribution”, Sankhyā Ser. A 32 (1970), 311-318. Zbl 225.62016.

[8] C. G. Khatri and K. C. S. Pillai, On the non-central distributions of two test criteria in multivariate analysis of variance, Ann. Math. Statist. 39 (1968), 215-226. MR 36\#2265. Zbl 155.27102.

[9] R. Khattree and R. D. Gupta, Some probability distributions connected with beta and gamma matrices, Comm. Statist. Theory Methods 21 (1992), no. 2, 369-390. MR 93b:62081. Zbl 800.62266.

[10] Y. Konno, Exact moments of the multivariate $F$ and beta distributions, J. Japan Statist. Soc. 18 (1988), no. 2, 123-130. MR 90f:62166. Zbl 669.62031.

[11] S. K. Mitra, A density-free approach to the matrix variate beta distribution, Sankhyā Ser. A 32 (1970), 81-88. MR 45\#2832. Zbl 203.51906.

[12] I. Olkin, Note on 'the Jacobians of certain matrix transformations useful in multivariate analysis', Biometrika 40 (1953), 43-46. MR 15,94b. Zbl 052.14904.

[13] I. Olkin and H. Rubin, Multivariate beta distributions and independence properties of the Wishart distribution, Ann. Math. Statist. 35 (1964), 261-269. MR 28\#3511.

[14] W. Y. Tan, Note on the multivariate and the generalized multivariate beta distributions, J. Amer. Statist. Assoc. 64 (1969), 230-241. MR 39\#2244. Zbl 175.17307.

ArJun K. Gupta: Department of Mathematics and statistics, Bowling Green State UNIVERSITY, BOWLING GREEN, OHIO 43403-0221, USA

Daya K. Nagar: Departamento de Mathemáticas, Universidad de ANTioquia, MedelLÍN, A.A. 1226, Colombia 


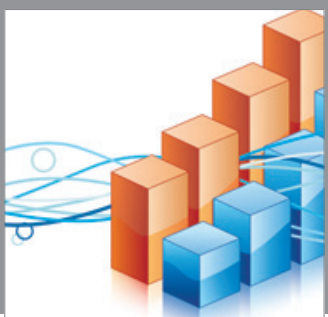

Advances in

Operations Research

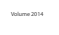

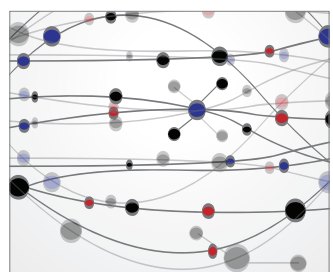

\section{The Scientific} World Journal
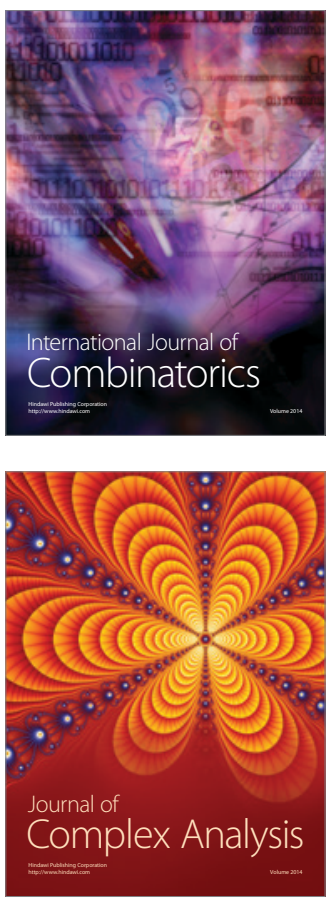

International Journal of

Mathematics and

Mathematical

Sciences
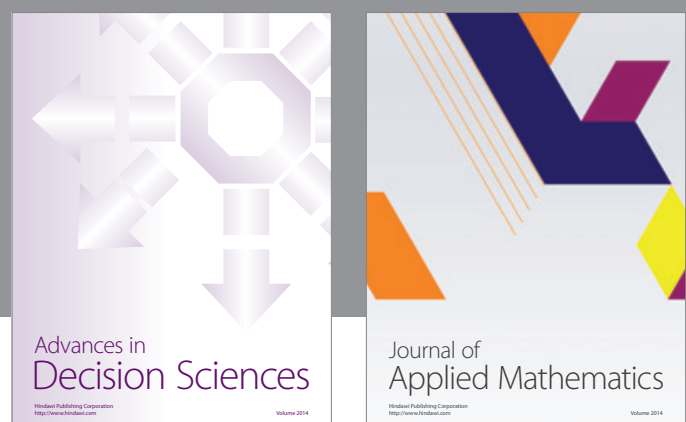

Journal of

Applied Mathematics
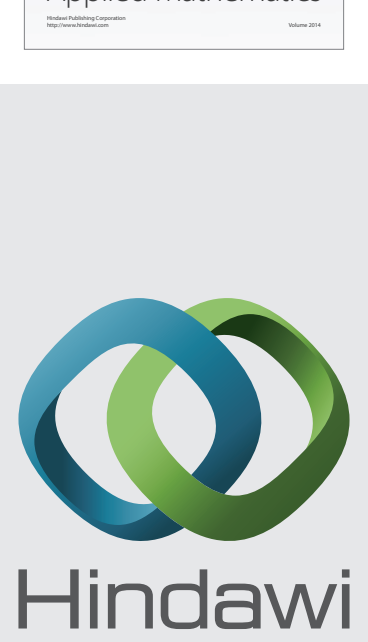

Submit your manuscripts at http://www.hindawi.com
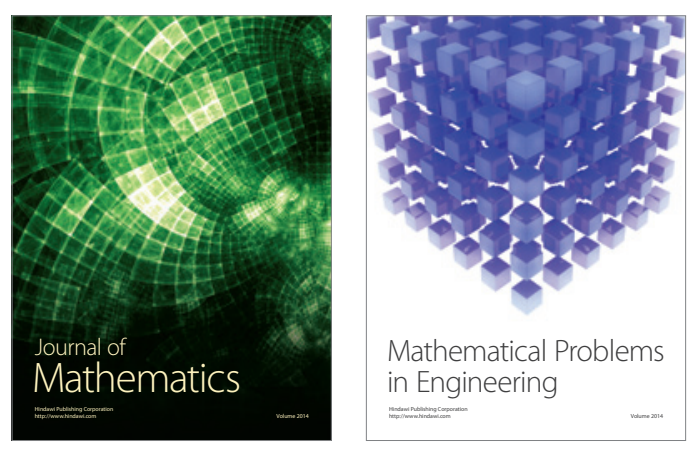

Mathematical Problems in Engineering
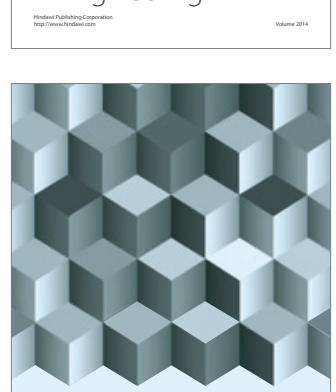

Journal of

Function Spaces
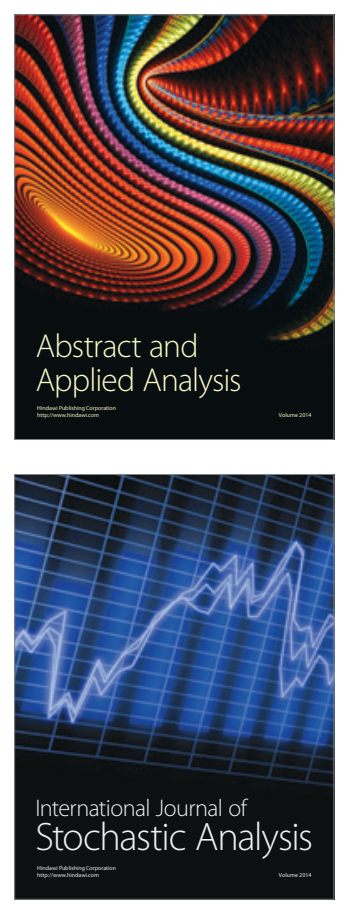

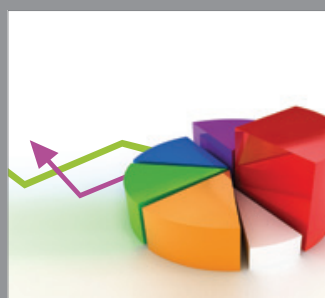

ournal of

Probability and Statistics

Promensencen
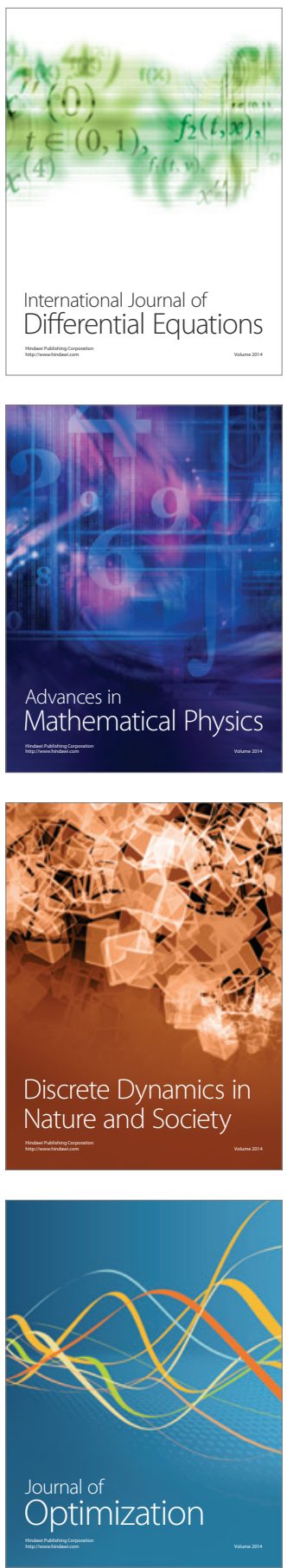